\title{
The Blindness Hypothesis and Individual Level Predicates
}

\author{
Giorgio Magri \\ Massachusetts Institute of Technology
}

Predicates such as 'to be tall', 'to be related to Chomsky' or 'to know Latin', which intuitively denote permanent properties, are called individual-level predicates (henceforth: i-predicates); predicates such as 'to be available', 'to talk to Chomsky' or 'to study Latin', which denote properties not necessarily permanent, are called stage-level predicates (henceforth: s-predicates). An impressive list of grammatical facts have been pointed out that set the two classes of predicates apart. The overall picture is that s-predicates can do many more things than i-predicates can do: there are constructions where s-predicates are fine but i-predicates are not (e.g. temporal and locative modification, 'there'-construction, perception sentences, etc.) and there are readings which are available with s-predicates but absent with i-predicates (e.g. the existential reading for their bare plural subjects, the episodic reading, etc.); some examples will be considered below. A theory of i-predicates should account for why i-predicates cannot do the many things that s-predicates can do. In Section 2 , I'll sketch (the beginning of) a theory of i-predicates based on scalar implicatures. For instance, I'll suggest that the sentence '?John is tall after dinner' sounds odd because it triggers the implicature that John is not tall also in non-after-dinner times, which mismatches with the common knowledge that tallness is a permanent property; see Percus (1997) and Maienborn (2004) among others, for a similar proposal. The main goal of Section 2 is to show that this simple idea extends also to less obvious cases, such as the restrictions on the readings of bare plural subjects of i-predicates. In order for the account to work, the following assumption is needed: that the algorithm for the computation of scalar implicatures is a purely logical algorithm, blind to common knowledge (the Blindness Hypothesis). Section 1 introduces this and some other background assumptions.

\section{The Blindness Hypothesis and Other Background Assumptions}

The goal of this Section is to develop an account for the oddness of the sentences (1), (2) and (3b), which have nothing to do with i-predicates. ${ }^{1}$

a. ?Some (of the) parents of the victim got married in church.

b. ?John was found dead with some of his hands tied together.

a. ?Every eye of the victim is blue.

b. ?Both eyes of the victim are blue.

I wish to thank Gennaro Chierchia, Danny Fox and Irene Heim.

${ }^{1}$ The example in (3) is a slight variant of an example discussed in Percus (2001). 
(3) Scenario: five different competitions were held separately, monday through friday; both Q and A know that the same guy won all five of them; Q wants to know who this amazing guy is.

a. Q: Who is the guy who always won?

b. A: ?Each day, a firemen won.

To illustrate the intuitive idea of the account which I'll pursue, consider for instance sentence (1a), repeated below in (4a).

(4) a. ?Some parents of the victim got married in church.

b. The parents of the victim got married in church.

I suggest that the oddness of sentence (4a) can be accounted for by means of the informal piece of reasoning in (5).

(5) a. On the one hand, sentence (4a) triggers the scalar implicature that the speaker is not in a position to utter $(4 \mathrm{~b})$, i.e. that he cannot presume that all the parents of the victim got married in church.

b. On the other hand, it follows from common knowledge that people have only two parents, hence (4a) cannot be true without (4b) being true too.

c. In conclusion, the oddness of (4a) follows from the mismatch between (5a) and (5b).

The piece of reasoning in (5) was first suggested in the literature by Hawkins (1991), and then discussed by Heim (1991). In the rest of this Section, I'll recast this piece of reasoning in more formal terms and apply it to (1), (2) and (3). In the next Section, I will apply it to various properties of i-predicates.

\subsection{On the Oddness of Sentences (1)}

Each sentence is associated with both a plain meaning and a strengthened meaning, namely the plain meaning enriched with its scalar implicatures. Following much recent literature, I will assume that the strengthened meaning of a sentence $\varphi$ is obtained by applying to $\varphi$ an exhaustivity operator $\operatorname{EXH}$, i.e. that it is $\operatorname{EXH}(\varphi)$. Many of the definitions of the exhaustivity operator EXH share the structure in (6): $\operatorname{EXH}(\varphi)$ asserts $\varphi$ and furthermore negates a bunch of alternatives $\psi$, namely all the alternatives $\psi$ in the set $\mathscr{E} x c l(\varphi)$ of the excludable alternatives given $\varphi$.

$$
\operatorname{EXH}(\varphi)=\varphi \wedge \bigwedge\{\neg \psi \mid \psi \in \mathscr{E} x c l(\varphi)\}
$$

The set $\mathscr{E} x c l(\varphi)$ of excludable alternatives wrt $\varphi$ is a subset of the set $\mathscr{A} l t(\varphi)$ of the scalar alternatives of $\varphi$, usually defined as in (7).

(7) The set $\mathscr{A} l t(\varphi)$ contains all and only those $\psi$ 's that can be obtained from $\varphi$ by replacing one or more scalar items in $\varphi$ with their Horn-mates.

Thus, these various definitions of the exhaustivity operator differ in how they carve $\mathscr{E} x c l(\varphi)$ out of $\mathscr{A} l t(\varphi)$. Here, I'll adopt the definition in (8), from Fox (2006). 
a. A subset $X=\left\{\psi_{1}, \psi_{2}, \ldots\right\}$ of the set $\mathscr{A} l t(\varphi)$ is called an innocently excludable subset wrt $\varphi$ iff $\varphi \wedge \neg \psi_{1} \wedge \neg \psi_{2} \wedge \ldots$ is not a contradiction.

b. A subset $X \subseteq \mathscr{A} l t(\varphi)$ is called a maximal innocently excludable subset wrt $\varphi$ iff there are no innocently excludable supersets of $X$ in $\mathscr{A} l t(\varphi)$.

c. The intersection of all maximal innocently excludable subsets of $\mathscr{A} l t(\varphi)$ wrt $\varphi$ is denoted by $\mathscr{E} x c l(\varphi)$ and called the set of excludable alternatives.

The insight formalized by (8) is that the strengthened meaning $\operatorname{EXH}(\varphi)$ asserts $\varphi$ and excludes as many scalar alternatives $\psi$ 's as can be excluded in a non-arbitrary way without getting a contradiction; see Fox (2006) for discussion and examples.

Consider sentence $\varphi$ in (9a). By (7), the set $\mathscr{A} l t(\varphi)$ of scalar alternatives of $\varphi$ contains the three alternatives $\psi_{\mathrm{FA}}, \psi_{\mathrm{NS}}$ and $\psi_{\mathrm{NA}}$ in $(9 \mathrm{~b})$, under the standard assumption that 〈'some', 'all' $\rangle$ and 〈'few', 'none' $\rangle$ are Horn-scales. Of course, $\psi_{\mathrm{FA}}$ is not excludable given $\varphi$, since $\psi_{\mathrm{FA}}$ is entailed by $\varphi$. Thus, the set of excludable alternatives is $\mathscr{E} x \operatorname{cl}(\varphi)=\left\{\psi_{\mathrm{NS}}, \psi_{\mathrm{NA}}\right\}$, since these two alternatives $\psi_{\mathrm{NS}}$ and $\psi_{\mathrm{NA}}$ can be negated consistently with $\varphi$. Thus, the strengthened meaning of sentence $\varphi$ is predicted to be $(9 \mathrm{c})$, namely "few boys did some of the readings and some boy did some of the readings and some boy did all of the readings." This result is of course wrong: an utterance of $\varphi$ does not in any way commit to the existence of a boy who has done all of the readings.
a. $\varphi=$ Few boys did some of the readings.
b. i. $\psi_{\mathrm{FA}}=$ Few boys did all of the readings.
ii. $\psi_{\mathrm{NS}}=$ No boys did some of the readings.
iii. $\psi_{\mathrm{NA}}=$ No boys did all of the readings.
c. $\operatorname{EXH}(\varphi)=\varphi \wedge \neg \psi_{\mathrm{NS}} \wedge \neg \psi_{\mathrm{NA}}$.

In order to get rid of this wrong inference, we need to get rid of the alternative $\psi_{\mathrm{NA}}$. Note the following crucial fact: while the alternative $\psi_{\mathrm{NS}}$ (asymmetrically) entails $\varphi$, the alternative $\psi_{\mathrm{NA}}$ does not, since it is logically independent of $\varphi$. I thus suggest that the set $\mathscr{E} x c l(\varphi)$ of alternatives excludable given $\varphi$ should only contain those scalar alternatives which asymmetrically entail $\varphi .^{2}$ In other words, I submit that clause (a) of definition (8) should be modified as follows.

a. A subset $X=\left\{\psi_{1}, \psi_{2}, \ldots\right\}$ of scalar alternatives of $\varphi$ is called an innocently excludable subset wrt $\varphi$ iff $\varphi$ asymmetrically entails each $\psi \in X$ and furthermore $\varphi \wedge \neg \psi_{1} \wedge \neg \psi_{2} \wedge \ldots$ is not a contradiction.

This little modification of the original definition (8) will play a role only in 2.3.

The definition of the exhaustivity operator EXH that I have in place at this point makes use of the two notions of asymmetric entailment and contradictoriness. Both notions can be defined in terms of the notion of entailment. To complete the definition of the exhaustivity operator, I thus need to spell out the relevant notion of

\footnotetext{
${ }^{2}$ Or, alternatively, which are not independent of $\varphi$. See also Fox's (2006) footnote 35 for an alternative solution, fully compatible with the proposal in this paper. I have chosen the option in (10) only for the sake of explicitness.
} 
entailment. Let $\mathscr{W}_{\text {ck }}$ be the subset of the set $\mathscr{W}$ of all possible worlds where common knowledge holds, i.e. where people have only two parents, two eyes of the same color, etc. The notion of entailment can thus be spelled out in two different ways: as logic entailment (i.e. $\rightarrow_{\mathscr{W}}$ ) or as entailment given common knowledge $\mathscr{W}_{\mathrm{ck}}$ (i.e. $\left.\rightarrow \mathscr{W}_{\mathrm{ck}}\right)$. The definitions are provided in (11).

(11) For any two propositions $\varphi, \psi$ :

a. $\psi$ logically entails $\varphi$ (i.e. $\psi \rightarrow_{\mathscr{W}} \varphi$ ) iff $\psi \subseteq \varphi$.

b. $\psi$ entails $\varphi$ given common knowledge $\mathscr{W}_{\mathrm{ck}}\left(\right.$ i.e $\psi \rightarrow_{\mathscr{W}_{\mathrm{ck}}} \varphi$ ) iff $\psi \cap \mathscr{W}_{\mathrm{ck}} \subseteq \varphi$.

Which one of these two notions is the one relevant for the definition of the exhaustivity operator EXH? Here is my answer:

(12) The notion of entailment relevant for the definition of the exhaustivity operator EXH is that of logic entailment rather than that of entailment given common knowledge $\mathscr{W}_{\mathrm{ck}}$.

In other words, the computation of the strengthened meaning $\operatorname{EXH}(\varphi)$ is blind to common knowledge. I'll thus dub (12) as the Blindness Hypothesis (henceforth: $\mathrm{BH})$. See Fox and Hackl (2005) for independent evidence that the computation of scalar implicatures is indeed blind to common knowledge. Thus, by definition, the strengthened meaning cannot be a logical contradiction. This of course doesn't exclude the possibility of the strengthened meaning being a contradiction given common knowledge. What happens in this case? Here is my answer:

(13) For every sentence $\varphi$, if its strengthened meaning $\operatorname{EXH}(\varphi)$ (computed without taking common knowledge into account) is a contradiction given common knowledge (i.e. $\operatorname{EXH}(\varphi) \cap \mathscr{W}_{\mathrm{ck}}=\emptyset$ ), then the sentence $\varphi$ sounds odd.

For the time being, I present (12) and (13) as bare stipulations, and defer to future work a more through discussion of their status and their consequences.

I am now in a position to account for the oddness of the two sentences (1). For concreteness, consider sentence (1a), repeated as $\varphi$ in (14a).

a. $\varphi=$ ?Some parents of the victim got married in church.

b. $\psi=$ The parents of the victim got married in church.

Consider the alternative $\psi$ in (14b). Let me assume that 〈'some', 'the' $\rangle$ is a Hornscale; hence $\psi$ is a scalar alternative of $\varphi$. Of course, $\psi$ logically entails $\varphi$. But the opposite doesn't hold: in a world where the victim has three parents and only two of them got married in church, $\varphi$ would be true while $\psi$ false. Hence, $\psi$ logically asymmetrically entails $\varphi$. Thus, $\mathscr{E} x \operatorname{cl}(\varphi)=\{\psi\}$ and the strengthened meaning of $\varphi$ boils down to $\operatorname{EXH}(\varphi)=\varphi \wedge \neg \psi$, namely "some but not all of the parents of the victim got married in church." Of course, this strengthened meaning $\operatorname{EXH}(\varphi)$ is a contradiction given the piece of common knowledge that people have exactly two parents. In fact, consider an arbitrary world $w \in \mathscr{W}_{\mathrm{ck}}:$ if $\varphi$ is true in $w$, then two or more of the parents of the victim got married in church in $w$; since $w$ is compatible with common knowledge $\mathscr{W}_{\text {ck }}$, the victim has only two parents in $w$; hence, all of the 
parents of the victim got married in church in $w$, i.e. $\psi$ is true too in $w$. The oddness of sentence $\varphi$ is thus predicted by (13). Note the crucial role that the BH (12) plays in this piece of reasoning, as first pointed out by Heim (1991): if the strengthened meaning were computed using entailment relative to common knowledge, then $\psi$ could not count as an excludable alternative wrt $\varphi$, since $\varphi$ and $\psi$ are equivalent given the common knowledge that people have exactly two parents.

\subsection{On the Oddness of Sentence (3b)}

The oddness of (3b), as an answer to (3a), is intuitively due to the fact that (3b) somehow mismatches with the presupposition introduced by the question (3a), namely that a unique guy won each of the five competitions. Let me show how to capture this mismatch. In the literature on the pragmatics of question/answer pairs, one often finds the observation that sentence $\varphi$ in (15b), as an answer to (15a), suggests that the speaker doesn't know, for each individual fireman, whether Fred talked to him.

a. Who did Fred talk to?

b. $\varphi=$ He talked to some fireman.

This fact can be captured by assuming that the set of scalar alternatives of $\varphi$ is (16). For the sake of explicitness, let me assume that this set of alternatives is built by replacing the DP 'some fireman' in $\varphi$ with something like the definite description 'the fireman/men such and such' for all possible choices of 'such and such', which thus need to be Horn-mates.

$$
\begin{aligned}
& \mathscr{A} l t(\varphi)=\left\{\psi_{d(\cdot)} \mid d(\cdot) \in \mathscr{D}_{e}^{\mathscr{W}}\right\}, \\
& \text { where } \psi_{d(\cdot)}=\lambda w:[[\text { fireman }]]^{w}(d(w)) \cdot[[\text { talk }]]^{w}(\text { Fred }, d(w)) .
\end{aligned}
$$

Let me now apply this assumption to the case of our sentence (3b). Its LF is the one in (17a). This LF corresponds to the truth conditions $\varphi$ in (17b), namely that "for each day of the competition, there was a firemen who won on that day." Note that 'each day' has scope over 'a fireman' (i.e. $\forall_{t}>\exists_{x}$ ) in (17b).

a. [[each day] $[\lambda t[[$ a fireman] $[\lambda x[x$ won in $t]]]]]$.

b. $\varphi=\lambda w \cdot \forall_{t}\left[[[\text { day }]]^{w}(t) \rightarrow\left[\exists_{x}\left[[[\text { fireman }]]^{w}(x) \wedge[[\text { win }]]^{w}(x, t)\right]\right]\right.$.

Consider now the LF in (18a): since it is obtained from (17a) by replacing 'a firemen' by 'the fireman such and such' (abbreviated as 'the fireman $P$ '), then the LF (18a) is a scalar alternative of the LF (17a). The LF (18a) yields the truth conditions $\psi_{P}$ in (18b).

a. [[each day] $[\lambda t[$ [the fireman $P][\lambda x[x$ won in $t]]]]]$.

b. $\psi_{P}=\lambda w \cdot \forall_{t}\left[[[\text { day }]]^{w}(t) \rightarrow\left[[[\text { the fireman } P]]^{w} \in[[\text { win }]]^{w}(t)\right]\right]$.

Let's look at these alternatives $\psi_{P}$ in some more detail. Consider the formula $\beta$ in (19b), which is identical to $\varphi$ in (17b) only with the opposite scope relationship between 'every day' and 'a fireman'. The logical equivalence in (19) is easy to verify. 
(19) For every possible world $w: \alpha(w)=1$ iff $\neg \beta(w)=1$.

$$
\begin{aligned}
& \text { a. } \alpha \doteq \wedge\left\{\neg \psi_{P} \mid P\right\} . \\
& \text { b. } \beta \doteq \lambda w . \exists_{x}\left[[[\text { fireman }]]^{w}(x) \wedge \forall_{t}\left[[[\text { day }]]^{w}(t) \rightarrow[[\text { win }]]^{w}(x, t)\right]\right] .
\end{aligned}
$$

Let me now argue that the strengthened meaning $\operatorname{ExH}(\varphi)$ of the odd sentence $\varphi$ in (17b) is the one in (20), i.e. that each $\psi_{P}$ is excludable wrt $\varphi$. Note that each $\psi_{P}$ logically asimmetrically entails $\varphi$. Thus, under the assumption that there are no other relevant alternatives, it is sufficient to show that $\operatorname{EXH}(\varphi)$ in (20) is not a logical contradiction. And because of the logical equivalence in (19), it is sufficient to show that $\varphi \wedge \neg \beta$ is not a logical contradiction. And of course it is not, since there are possible worlds were different firemen have won on different days, where thus $\varphi$ is true but $\beta$ false.

$$
\operatorname{EXH}(\varphi)=\varphi \wedge \underbrace{\bigwedge\left\{\neg \psi_{P} \mid P\right\}}_{\alpha} .
$$

Note nonetheless that $\operatorname{EXH}(\varphi)=\varphi \wedge \neg \beta$ is a contradiction relative to the presupposition introduced by the question in (3a), namely that a single fireman has won on every day. The oddness of $\varphi$ in (3b) as an answer to (3a) thus follows from (13).

\subsection{On the Oddness of Sentences (2)}

The account I have in place so far doesn't quite work for the two sentences (2), since these two sentences express the strongest meaning among their alternatives. I'll suggest that their oddness is due to the fact that, nonetheless, they do not carry the strongest presupposition. To keep things simple, assume that presuppositional items denote partial functions of the right type. Hence, a sentence $\varphi$ containing a presuppositional item denotes a partial function from possible worlds into truthvalues. Let $\varphi_{\text {prs }}$ be the domain of such a function, namely the presupposition carried by $\varphi$. Assume that strengthening is performed also at the level of presupposition, i.e. that we can associate with each sentence $\varphi$ its strengthened presupposition $\operatorname{EXH}\left(\varphi_{\text {prs }}\right)$. The definition of the strengthened presupposition $\operatorname{EXH}\left(\varphi_{\text {prs }}\right)$ is identical to that of strengthened meaning given above, namely (21), where the set $\mathscr{E} x c l\left(\varphi_{\text {prs }}\right)$ is defined just as in (10), replacing $\varphi$ by $\varphi_{\mathrm{prs}}$ and $\psi$ by $\psi_{\mathrm{prs}}{ }^{3}$

$$
\operatorname{EXH}\left(\varphi_{\text {prs }}\right)=\varphi_{\text {prs }} \wedge \bigwedge\left\{\neg \psi_{\text {prs }} \mid \psi_{\text {prs }} \in \mathscr{E} x c l\left(\varphi_{\text {prs }}\right)\right\}
$$

Of course, I want to maintain that the Blindness Hypothesis holds also for the computation of strengthened presuppositions, as stated in (22) which I'll refer to as the $\mathrm{BH}^{\prime}$. The $\mathrm{BH}^{\prime}$ suggests the variant of assumption (13) stated in (23). The two hypotheses (22) and (23) are an old idea, suggested by Heim (1991) and then developed by Sauerland (2003a) under the name of Maximize Presupposition.

\footnotetext{
${ }^{3}$ I am assuming that the set of scalar alternatives used to compute the strengthened presupposition and the strengthened meaning is the same. The only difference is that in the former case we look at the presuppositions of those alternatives while in the latter we look at their meaning.
} 
(22) The notion of entailment relevant for the computation of the strengthened presupposition $\operatorname{EXH}\left(\varphi_{\text {prs }}\right)$ is that of logic entailment " $\rightarrow$ " rather than that of entailment relative to common knowledge " $\rightarrow_{\mathscr{W}_{\mathrm{ck}}}$ ".

(23) For every sentence $\varphi$, if its strengthened presupposition $\operatorname{EXH}\left(\varphi_{\text {prs }}\right)$ (computed without taking common knowledge into account) is a contradiction given common knowledge (i.e. $\operatorname{EXH}\left(\varphi_{\text {prs }}\right) \cap \mathscr{W}_{\mathrm{ck}}=\emptyset$ ), then $\varphi$ sounds odd.

I am now in a position to account for the oddness of the two sentences (2). For concreteness, consider (2a), repeated as $\varphi$ in (24a). ${ }^{4}$

a. $\varphi=$ ?Every eye of the victim is blue.

b. $\psi=$ The eyes of the victim are blue.

Consider the alternative $\psi$ in (24b). Let me assume that 〈'every', 'the' $\rangle$ is a Hornscale; hence, $\psi$ is a scalar alternative of $\varphi$. Let us thus briefly pause on the proper semantics of sentence $\psi$. Plural predication with distributive predicates, as in the case of $\psi$, requires the predicate to be operated upon by the distributive operator Dist. Following for instance Link (1983), I take the function Dist $\left([[\mathrm{VP}]]^{w}\right)$ to be true of a plurality $x$ iff the property $[[\mathrm{VP}]]^{w}$ holds of all the atomic parts $y$ of $x$ (i.e. $y \leq_{\mathrm{AT}} x$ ). Thus, $\varphi$ and $\psi$ end up having the same meaning. Nonetheless, they have different presuppositions. In fact, the distributivity operator Dist does something more: it also introduces the presupposition that the property [[VP] $]^{w}$ either holds of all the atomic parts $y$ of $x$ or else it does not hold of any of them. This presupposition is called the homogeneity presupposition in Gajewski (2005) and was first discussed in Fodor (1970). Thus, the proper semantics of the distributive operator Dist is that in (25). The homogeneity presupposition can be detected by means of negation: the sentence 'Sue didn't see the boys' conveys that Sue didn't see any of the boys, which differs from the plain meaning (namely that "Sue didn't see every boy") but does follow from it plus the homogeneity presupposition.

$\operatorname{Dist}\left([[\mathrm{VP}]]^{w}\right)=\lambda x: \operatorname{YES}^{w}(x) \vee N O^{w}(x) \cdot \forall y\left[y \leq_{\mathrm{AT}} x \rightarrow[[\mathrm{VP}]]^{w}(y)\right]$.

a. $Y E S^{w}(x)=1$ iff $[[\mathrm{VP}]]^{w}(y)$ for every $y$ such that $y \leq_{\mathrm{AT}} x$.

b. $N O^{w}(x)=1$ iff $\neg[[\mathrm{VP}]]^{w}(y)$ for every $y$ such that $y \leq_{\mathrm{AT}} x$.

\footnotetext{
${ }^{4}$ One might be tempted to account for the oddness of (24a) by assuming that 'every' carries an anti-duality presupposition on its restrictor, which in the case of sentence (24a) is violated in every world in $\mathscr{W}_{\mathrm{ck}}$. Sauerland (2003b) offers the following contrast as an argument against this account.

(i) Scenario: several candidates applied; some have written only one paper, others two, and the rest have written more than two. The selection committee decides:

a. ?Every candidate should send [the paper of his].

b. Every candidate should send [every paper of his].

The oddness of sentence (a) is immediately accounted for under the assumption that the uniqueness presupposition of the definite 'the paper of his' is projected universally; if 'every paper of his' had an anti-duality presupposition, it would be predicted to project universally as well and sentence (b) would be predicted to suffer from presupposition failure just as sentence (a), contrary to facts.
} 
Let's now go back to $\varphi$ and $\psi$ in (24). By (25), $\psi$ bears the homogeneity presupposition $\psi_{\text {prs }}$ in (26b), according to which the eyes of the victim are either both blue or else none of them is. No such presupposition is carried by $\varphi$, as stated in (26a). Here, I am ignoring the existence presupposition.
a. $\varphi_{\mathrm{prs}}=\mathscr{W}$.
b. $\psi_{\mathrm{prs}}=Y E S \cup N O$.

i. $Y E S=\{w \mid$ all eyes of the victim are blue in $w\}$.

ii. $N O=\{w \mid$ none of the eyes of the victim is blue in $w\}$.

Of course, $\psi_{\text {prs }}$ asymmetrically entails $\varphi_{\text {prs }}$. Under the assumption that there are no other excludable presuppositions, $\mathscr{E} x \operatorname{cl}\left(\varphi_{\text {prs }}\right)=\left\{\psi_{\text {prs }}\right\}$ and the strengthened presupposition of $\varphi$ boils down to $\operatorname{EXH}\left(\varphi_{\text {prs }}\right)=\varphi_{\text {prs }} \wedge \neg \psi_{\text {prs }}=\neg \psi_{\text {prs }}$. This strengthened presupposition is a contradiction given the common knowledge that people have eyes of the same color, since $\psi_{\text {prs }}$ follows from such a common knowledge. The oddness of sentence (24a) is thus predicted by (23). Note again the crucial role that the $\mathrm{BH}^{\prime}$ (22) plays in this piece of reasoning: if the strengthened presupposition were computed using entailment relative to common knowledge, then $\psi_{\text {prs }}$ could not count as an excludable alternative wrt $\varphi_{\text {prs }}$, since $\varphi_{\text {prs }}$ and $\psi_{\text {prs }}$ are equivalent given the common knowledge that people have eyes of the same color.

\section{Application to Individual-Level Predicates}

Let me assume that there is no relevant grammatical difference between s- and ipredicates, contra Kratzer (1995), Diesing (1992), Chierchia (1995). For example, there is no difference with respect to the reconstruction possibilities of their subjects or with respect to their argumental structure, namely:

(27) Both 'tall' and 'available' have a davidsonian argument which, for the sake of simplicity, I will naïvely take to range over times $t \in \mathscr{T}$.

What is the relevant difference between s- and i-predicates, then? I assume that all that's special about an i-predicate such as 'tall' is (28), which crudely captures the intuition that i-predicates denote permanent properties.

(28) For every world $w \in \mathscr{W}_{\mathrm{ck}}$ compatible with common knowledge, for every individual $d \in \mathscr{D}_{e}$ and for every time $t^{\prime} \in \mathscr{T}$, if $[[\operatorname{tall}]]^{w}\left(d, t^{\prime}\right)$, then $[[\operatorname{tall}]]^{w}(d, t)$ for every time $t$ in the life $\operatorname{span} \operatorname{in}^{w}(d, \cdot)$ of $d$ in $w$.

As noted at the beginning, a theory of i-predicates should account for why i-predicates cannot do the many things that s-predicates can do. I want to suggest that, under assumptions (27) and (28), such a theory is a theorem of the more general theory of oddness sketched in Section 1. Namely, that those many things that i-predicates cannot do would correspond to truth-conditions whose strengthened meaning (or strengthened presupposition), computed accordingly to the $\mathrm{BH}$ (or the $\mathrm{BH}^{\prime}$ ), would be a contradiction given common knowledge (28), and are thus ruled out by (13) 
(or (23)). In this Section, I consider three such things that i-predicates cannot do, and show that they can be accounted for in the same way I have accounted above for the oddness of (1), (2) and (3b), respectively.

\subsection{Locative and Temporal Modification}

Contrary to s-predicates, i-predicates (often) ban locative and temporal modification, as shown by the different status of the two sentences (29).

a. John is available after dinner.

b. ?John is tall after dinner.

The truth conditions of the fine sentence (29a), with the habitual s-predicate 'available', are usually taken to be those in (30a). These truth-conditions contain a generic operator $\mathrm{GEN}_{t}$ which ranges over times and takes two arguments. Its first argument, called the restrictive clause, is the set of those times which satisfy the contextually supplied restriction $\mathbf{C}$ and furthermore are after-dinner times. The second argument of the generic operator, called its nuclear scope, is the set of those times at which John is available. Thus, (30a) says that "for every after-dinner time $t$ which satisfies $\mathbf{C}$, John is available at that time $t$." Under the assumption that there is no difference between s- and i-predicates, the same truth-conditions should be available also for sentence (29b), with the i-predicate 'tall'. I have written them down as $\varphi$ in (30b). Thus, the problem of accounting for the oddness of (29b) boils down to the problem of ruling out $\varphi$ in (30b). Let me now show that these truth conditions $\varphi$ in (30b) can be ruled out in the same way as I have ruled out the odd sentences (1) in Section 1.

$$
\begin{aligned}
& \text { a. } \lambda w \cdot \operatorname{GEN}_{t}\left[\mathbf{C}^{w}(j, t) \wedge[[\text { after-dinner }]]^{w}(t)\right]\left[[[\text { available }]]^{w}(j, t)\right] . \\
& \text { b. } \varphi=\lambda w \cdot \operatorname{GEN}_{t}\left[\mathbf{C}^{w}(j, t) \wedge[[\text { after-dinner }]]^{w}(t)\right]\left[[[\text { tall }]]^{w}(j, t)\right] .
\end{aligned}
$$

Consider (31a), with the truth-conditions $\psi$ in (31b). Note that $\psi$ is obtained from $\varphi$ by modifying the restrictive clause of the generic operator. Let me assume that the restrictive clause can be modified without falling out of the set of scalar alternatives of $\varphi$. This assumption is supported by the fact that sentence (29a) does indeed trigger the implicature that it is false (or that the speaker doesn't know) that John is available in non-after-dinner times. Thus, $\psi$ is a scalar alternative of $\varphi$.

a. John is tall.

b. $\psi \doteq \lambda w \cdot \operatorname{GEN}_{t}\left[\mathbf{i n}^{w}(j, t)\right]\left[[[\operatorname{tall}]]^{w}(j, t)\right]$.

Of course, $\psi$ logically entails $\varphi$. Furthermore, $\varphi$ does not logically entail $\psi$ : in fact, consider a world (not in $\mathscr{W}_{\mathrm{ck}}$ ) where John is tall only after dinner and note that $\varphi$ is true but $\psi$ false in such a world. Under the assumption that $\psi$ is the only relevant alternative ${ }^{5}$ of $\varphi, \mathscr{E} x c l(\varphi)=\{\psi\}$ and the strengthened meaning of $\varphi$ boils down to $\operatorname{EXH}(\varphi)=\varphi \wedge \neg \psi$, namely that "John is tall only after-dinner." Of course,

\footnotetext{
${ }^{5}$ This is not quite true, since I should consider any possible modification of the restrictive clause; it is straightforward to check that this simplification doesn't affect the proposal.
} 
this strengthened meaning is a contradiction given the common knowledge $\mathscr{W}_{c k}$ in (28): if John is tall at after-dinner times in $w$ and if $w$ is in $\mathscr{W}_{\text {ck }}$, then John is tall through out his entire life span in $w$. Thus, $\varphi$ in (30b) is ruled out by (13). ${ }^{6}$

\subsection{Existential Bare Plural Subjects}

The bare plural subject (henceforth: BPS) 'firemen' of the s-predicate 'available' in (32a) admits both the generic and the existential reading; the BPS of the i-predicate 'tall' in (32b) instead lacks the existential reading and only allows the generic one.
a. Firemen are available.
$\exists$-BPS, GEN-BPS
b. Firemen are tall.
* $\exists$-BPS, GEN-BPS

Let me assume that the truth-conditions for the existential reading of the BPS 'firemen' in (32a) are those in (33c) (where $\left.\mathbf{C}^{w}([\text { firemen }]]^{w}, t\right)$ is some contextually relevant set of times where firemen are present), namely that "for every time $t$ which satisfies $\mathbf{C}$, there is a fireman $x$ who is available at $t$." Note that the generic operator $\mathrm{GEN}_{t}$ has wide scope over the existential operator $\exists_{x}$ in (33c). I assume that this scope configuration is the only possible one. I take this to be an instance of a more general property of existential bare plurals: that they always select the narrowest possible scope; see Carlson (1977). That $\operatorname{GEN}_{t}>\exists_{x}$ is the only scope configuration, follows from any proper theory of bare plurals, such as the Heimian semantics adopted in Diesing (1992) or the semantics developed in Chierchia $(1995,1998)$. To develop my proposal, I need the latter; here is a sketch.

$$
\begin{aligned}
& \text { a. }[\text { firemen }[\lambda x[\operatorname{GEN}[x \text { available }]]]] . \\
& \text { b. } \lambda w \cdot[[\text { firemen }]]^{w} \in \lambda x \cdot \operatorname{GEN}_{t}\left[\mathbf{C}^{w}(x, t)\right]\left[[[\text { available }]]^{w}(x, t)\right] . \\
& \text { c. } \lambda w \cdot \operatorname{GEN}_{t}\left[\mathbf{C}^{w}\left([[\text { firemen }]]^{w}, t\right)\right]\left[\exists_{x}\left[[[\text { firemen }]]^{w}(x) \wedge[[\text { available }]]^{w}(x, t)\right]\right] .
\end{aligned}
$$

Assume that the LF of our sentence (32a) is (33a), which is straightforwardly mapped onto the truth-conditions (33b). Assume that bare plurals denote kinds. What happens when the predicate $[\text { available }]^{w}$ is saturated with the kind $\left[\right.$ firemen] ${ }^{w}$ ? Since $[$ available $]]^{w}$ is a predicate true of ordinary individuals and 'firemen' denotes a kind, the computation is stuck. Chierchia suggests that the mismatch is solved by means of the type shifter Derived Kind Predication $\langle\cdot\rangle_{\mathrm{DKP}}$ in (34). The existential quantification over firemen in (33c) is brought about by resorting to $\langle\cdot\rangle_{\mathrm{DKP}}$ in order to solve the mismatch between the kind $[\text { firemen }]^{w}$ and the predicate of ordinary individuals $[$ available $]]^{w}$. Just as any other type shifting device, $\langle\cdot\rangle_{\mathrm{DKP}}$ is only triggered by a mismatch. In other words, $\langle\cdot\rangle_{\mathrm{DKP}}$ is only triggered in the very

\footnotetext{
${ }^{6}$ Note that i-predicates do not always ban temporal modification: although sentence (29b) with the temporal modifier 'after dinner' sounds odd, a sentence such as 'John knows Latin since he was fifteen', with the temporal modifier 'since he was fifteen', sounds fine. This contrast is difficult to derive in frameworks which attribute the oddness of (29b) to some syntactic peculiarity of ipredicates, such as the ones by Kratzer (1995) and Chierchia (1995). However, this contrast is predicted by the account just sketched: the strengthened meaning of the latter sentence, namely that "John knows Latin only since he was fifteen", is compatible with common knowledge, contrary to the strengthened meaning of sentence (29b), namely that "John is tall only after dinner."
} 
embedded position at which we attempt to apply the bare plural [[firemen] $]^{w}$ to the predicate $[$ available $]]^{w}$. Thus, existential bare plurals always have the narrowest possible scope because of the "last resort" nature of $\langle\cdot\rangle_{\mathrm{DKP}}$.

(34) For every predicate of ordinary individuals $P$ :

$\langle P\rangle_{\text {DKP }} \doteq \lambda x_{k} \cdot \exists y_{o}\left[y_{o} \leq x_{k} \wedge P\left(y_{o}\right)\right]$

where: $x_{k}$ is a variable over kinds, $y_{o}$ is a variable over ordinary individuals and $\leq$ is the relation which holds between a kind and one of its instances.

Under the assumption that there are no grammatical differences between s- and ipredicates, the same LF and truth-conditions should be available also for sentence (32b), as stated in (35). Thus, the problem of accounting for the lack of the existential reading of the BPS 'firemen' of the i-predicate 'tall' in (32b) boils down to the problem of ruling out $\varphi$ in (35c), with narrow scope existential quantification over firemen. Note that these truth conditions are formally analogous to those in (17b) in Section 1. Let me thus argue that these truth conditions $\varphi$ in (35b) can be ruled out in the same way I have ruled out those in (17b).

$$
\begin{aligned}
& \text { a. }[\text { firemen }[\lambda x[\operatorname{GEN}[x \text { tall }]]]] . \\
& \text { b. } \lambda w \cdot[[\text { firemen }]]^{w} \in \lambda x \cdot \operatorname{GEN}_{t}\left[\mathbf{C}^{w}(x, t)\right]\left[[[\text { tall }]]^{w}(x, t)\right] . \\
& \text { c. } \varphi=\lambda w \cdot \operatorname{GEN}_{t}\left[\mathbf{C}\left([[\text { firemen }]]^{w}, t\right)\right]\left[\exists_{x}\left[[[\text { firemen }]]^{w}(x) \wedge[[\text { tall }]]^{w}(x, t)\right]\right] .
\end{aligned}
$$

Let me assume that 〈'firemen', 'the firemen such and such'〉 is a Horn-scale. Consider the LF in (36a): since it is obtained from the LF in (35a) by replacing 'firemen' with 'the firemen such and such' (here abbreviated as 'the firemen $P$ '), the LF (36a) is a scalar alternative of the LF (35a). The LF (36a) yields the truth-conditions in (36b), where the default distributivity operator Dist has been used to perform distributive predication. By virtue of the homogeneity presupposition of Dist in (25), these truth conditions can be made explicit as in (36c), where I have used the shorthand $[$ the firemen $P]]^{w} \doteq d(w)$.

a. [[the firemen $P][\lambda x[\operatorname{GEN}[x$ tall] $]]]$.

b. $\lambda w \cdot[[\text { the firemen } P]]^{w} \in \operatorname{Dist}\left(\lambda x \cdot \operatorname{GEN}_{t}\left[\operatorname{in}^{w}(x, t)\right]\left[[[\operatorname{tall}]]^{w}(x, t)\right]\right)$.

c. $\psi_{P} \doteq \lambda w: \operatorname{YES}(w) \vee N O(w) \cdot \bigwedge_{x \leq \mathrm{AT} d(w)} \operatorname{GEN}_{t}\left[\operatorname{in}^{w}(x, t)\right]\left[[[\operatorname{tall}]]^{w}(x, t)\right]$

i. $\operatorname{YES}(w)=1$ iff $\operatorname{GEN}_{t}\left[\operatorname{in}^{w}(x, t)\right]\left[[[\operatorname{tall}]]^{w}(x, t)\right]$ for every $x$ s.t. $x \leq_{\mathrm{AT}} d^{w}$

ii. $N O(w)=1$ iff $\neg \operatorname{GEN}_{t}\left[\operatorname{in}^{w}(x, t)\right]\left[[[\operatorname{tall}]]^{w}(x, t)\right]$ for every $x$ s.t. $x \leq_{\mathrm{AT}} d^{w}$

Let's consider these $\psi_{P}$ 's in some more detail. In particular, let's consider $\beta$ in (37b), which is roughly as $\varphi$ in (35b) only with the opposite scope configuration $\exists_{x}>\mathrm{GEN}_{t}$, and let me establish the logical equivalence stated in (37). Let me show that, if $\neg \beta(w)=1$, then $\alpha(w)=1$. By contradiction, suppose $\alpha(w) \neq 1$. Hence there is at least one $\psi_{P}$ which is either true or undefined in $w$. This in turn means that there must exist at least one firemen in $w$ who is tall throughout his entire life span in $w$. Hence, $\beta(w)=1$, contradicting the hypothesis. Let me now show that, if $\alpha(w)=1$, then $\neg \beta(w)=1$. Consider an arbitrary fireman in $w$, say John. Of course, there is some $P$ such that John $\leq_{\text {AT }}\left[[\text { the firemen } P]^{w}\right.$ (and, furthermore, such that 
$\psi_{P}$ asymmetrically entails $\varphi$ ). Note that $\psi_{P}$ must be false in $w$, because of the hypothesis $\alpha(w)=1$, i.e. $\psi_{P}(w)=0$. By virtue of the homogeneity presupposition (25) introduced by the distributive operator Dist, $\psi_{P}(w)=0$ iff for every $x$ such that $x \leq_{\mathrm{AT}}[$ the firemen $\left.P]\right]^{w}, x$ is not tall throughout his entire life span in $w$. Hence in particular the fireman John is not tall throughout his entire life span in $w$. Since this reasoning can be repeated for every other fireman in $w$ besides John, then $\neg \beta(w)=1$. The logical equivalence in (37) is thus established.

For every world $w \in \mathscr{W}: \alpha(w)=1$ iff $\neg \beta(w)=1$.
a. $\alpha=\bigwedge\left\{\neg \psi_{P} \mid \psi_{P} \stackrel{\mathrm{a}}{\rightarrow}_{\mathscr{W}} \varphi\right\}$.
b. $\beta=\lambda w \cdot \exists_{x}\left[[[\text { fireman }]]^{w}(x) \wedge \operatorname{GEN}_{t}\left[\operatorname{in}^{w}(x, t)\right]\left[[[\operatorname{tall}]]^{w}(x, t)\right]\right]$.

Let me now argue that the strengthened meaning of $\varphi$ is the one in (38), i.e. that all $\psi_{P}$ 's (which asymmetrically entail $\varphi$ ) are excludable wrt $\varphi$. Under the assumption that there are no other relevant alternatives besides $\psi_{P}$ 's, it suffices to argue that $\operatorname{EXH}(\varphi)$ in (38) is not a logical contradiction. And by virtue of the logical equivalence in (37), it suffices to exhibit a world where $\varphi \wedge \neg \beta$ is true.

$$
\operatorname{EXH}(\varphi)=\varphi \wedge \underbrace{\bigwedge\left\{\neg \psi_{P} \mid \psi_{P} \stackrel{\mathrm{a}}{\rightarrow}_{\mathscr{W}} \varphi\right\}}_{\alpha} .
$$

Consider the possible world $w$ represented in (39). Suppose that there are three firemen in $w$, namely $d_{1}, d_{2}$ and $d_{3}$. Their life span is represented with a thin segment. The thickened portion of each segment represents the portion of each life span throughout which the individual is tall in $w$. The long thin line at the top of the picture is the time axis $\mathscr{T}$; the thickened portion of it is the set of those times in the restrictive clause of formula $\varphi$, i.e. $\mathbf{C}^{w}\left([[\text { firemen }]]^{w}, \cdot\right)$. Note that $\varphi$ is true in this world $w$, because for each time $t$ in the set $\mathbf{C}^{w}$, either $d_{1}$ or $d_{2}$ or $d_{3}$ is tall at $t$. Note furthermore that $\beta$ is false in $w$, since no one of the three firemen $d_{1}, d_{2}, d_{3}$ is tall throughout his entire life span in $w$. Thus, $\operatorname{EXH}(\varphi)=\varphi \wedge \neg \beta$ is not a logical contradiction.

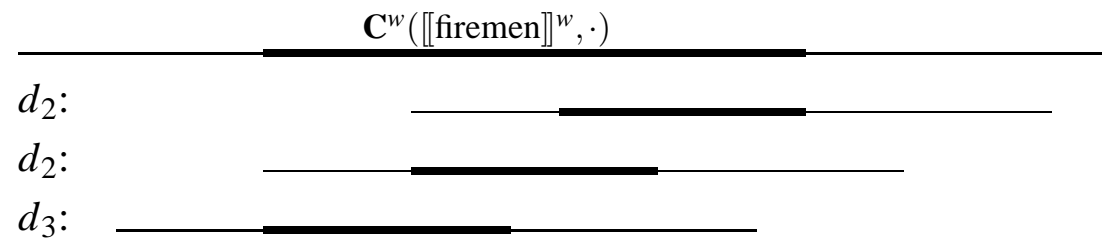

Note nonetheless that $\operatorname{EXH}(\varphi)=\varphi \wedge \neg \beta$ is a contradiction given the common knowledge $\mathscr{W}_{\text {ck }}$ in (28), precisely because no world such as the one in (39), in which firemen are tall only throughout a portion of their life span, can ever belong to $\mathscr{W}_{\text {ck }}$. In fact, suppose that $\operatorname{EXH}(\varphi)$ were true in a world $w \in \mathscr{W}_{\mathrm{ck}}$. Hence $\varphi$ must be true and $\beta$ false in $w$. Since $\varphi$ is true in $w$, then there must exist at least one fireman in $w$ who is tall in at least one instant in $w$, say John. Since $w$ is compatible with the common knowledge $\mathscr{W}_{\mathrm{ck}}$ in (28), John is tall throughout his entire life span in $w$. But then $\beta$ has got to be true too in $w$. Hence, $\operatorname{EXH}(\varphi)=\varphi \wedge \neg \beta$ is a contradiction given the common knowledge $\mathscr{W}_{c k}$ in (28). Let me wrap up: the existential 
reading for the BPS 'firemen' of the i-predicate 'tall' of sentence (32b) would correspond to truth conditions $\varphi$ in (35c), where existential quantification over firemen is embedded under the generic operator; because of this scope configuration, the strengthened meaning of these truth conditions $\varphi$ ends up being a contradiction given the common knowledge $\mathscr{W}_{\text {ck }}$ in (28); these truth conditions $\varphi$ are thus ruled out by (13) and the lack of the existential reading of the BPS of i-predicates is thus accounted for.

To conclude my discussion, let me consider the following variant (40) of sentence (32b), with the indefinite 'some firemen' instead of the PBS 'firemen'. This sentence (40) does of course admit the existential reading which is unavailable for (32b), namely "there is a firemen who is tall." Let me thus make sure that my proposal does not rule out the existential reading for this sentence (40) too.

(40) Some fireman is tall.

The crucial difference between this sentence (40) and the sentence (32b) I have considered so far, is the following: as noted above, (32b) is only compatible with narrow scope existential quantification over firemen; this is false for (40): its LF (41a) yields truth conditions (41b), trivially equivalent to $\beta$ in (37b), repeated in (41c), where existential quantification has scope over the generic operator.
a. [[A fireman] $[\lambda x$ [GEN $[x$ is tall $]]]]$.
b. $\lambda w \cdot[[\text { a fireman }]]^{w} \ni \lambda x \cdot \operatorname{GEN}_{t}\left[\mathbf{i n}^{w}(x, t)\right]\left[[[\operatorname{tall}]]^{w}(x, t)\right]$.
c. $\beta=\lambda w \cdot \exists_{x}\left[[[\text { fireman }]]^{w}(x) \wedge \operatorname{GEN}_{t}\left[\operatorname{in}^{w}(x, t)\right]\left[[[\operatorname{tall}]]^{w}(x, t)\right]\right]$.

Let me thus show that, precisely because of this different scope configuration, the strengthened meaning of $\beta$ in (41c) is not a contradiction given common knowledge. Under the assumption that 'some fireman' and 'the firemen such and such' are Horn-mates, each $\psi_{P}$ in (36c) is a scalar alternative of $\beta$ in (41c) (and, furthermore, each $\psi_{P}$ asymmetrically entails $\beta$ ). Note that in the case of $\beta$, contrary to the case of $\varphi$ in (35c), it is not possible to exclude all such $\psi_{P}$, since $\bigwedge \neg \psi_{P}$ is logically equivalent to $\beta$. Furthermore, there is not any single $\psi_{P}$ that can be left out in a non-arbitrary way. Thus, according to (8), no such $\psi_{P}$ will ever make it into the set of alternatives excludable given $\beta$ and thus there is no way that the strengthened meaning of $\beta$ can happen to be a contradiction given common knowledge.

\subsection{Existential Bare Plural Subjects in the Scope of a Universal Operator}

Fox (1995) observes that, quite surprisingly, the existential reading of the BPS of an i-predicate becomes available when the BPS is in the scope of a universal operator (or, more generally, in the scope of an operator which does not commute with the existential BPS). Here is one of his examples:

a. Jewish women are related to Chomsky. $* \exists$-BPS

b. Jewish women are related to every Jewish man.

$\sqrt{ } \exists$-BPS

As expected, the BPS 'Jewish women' in (42a) does not admit the existential reading. In sentence (42b), the definite 'Chomsky' has been replaced by the universal 
DP 'every Jewish man' and surprisingly the BPS 'Jewish women' admits the existential reading, i.e. the sentence can mean that "for every Jewish man there is a Jewish woman who is related to him." I'll now show that this contrast follows from the proposal developed so far.

Of course, the existential reading of the BPS of (42a) is ruled out in the same way as the existential reading of the BPS of (32b), since the reasoning in 2.2 can be repeated with $[$ related to $]]^{w}(\cdot$, Chomsky, $t)$ in place of $[[\text { tall }]]^{w}(\cdot, t)$. Let's now see what happens when this same line of reasoning is applied to (42b). Consider its LF (43a), obtained by leaving 'Jewish women' in [Spec, IP] and by adjoining 'every Jewish man' to VP by QR. This LF yields the truth-conditions in (43b), which in turn are equivalent to $\varphi$ in (43c) through $\langle\cdot\rangle_{\mathrm{DKP}}$, as discussed above. These truth conditions $\varphi$ correctly capture the reading of sentence (42b) we are after. Thus, let me show that my proposal does not rule out these truth conditions $\varphi$.
a. [[Jewish women] [ $\lambda x$ [[every Jewish man] $\lambda y[x$ related to $y]]]]$.
b. $\lambda w \cdot \llbracket$ women $\left.\left.\rrbracket^{w} \in \lambda x \cdot[\llbracket \text { every man }]^{w} \ni \lambda y \cdot \operatorname{GEN}_{t}\left[\mathbf{C}^{w}(x, y, t)\right][\llbracket \text { related }]^{w}(x, y, t)\right]\right]$.
c. $\left.\left.\left.\varphi=\lambda w \cdot \llbracket[\text { every man }]^{w} \ni \lambda y \cdot\left[\operatorname{GEN}_{t}\left[\mathbf{C}(\llbracket \text { women }]^{w}, y, t\right)\right]\left[\exists_{x}[\llbracket \text { women }]^{w}(x) \wedge \llbracket \text { related }\right]^{w}(x, y, t)\right]\right]\right]$.

By replacing 'Jewish women' with 'the Jewish women such and such' in (43a), we get (44a). The latter yields the truth conditions in (44b), where the distributive operator Dist has to be used in order to perform distributive predication. Truth conditions (44b) are in turn equivalent to $\psi_{P}$ in (44c), due to the homogeneity presupposition (25) of Dist (I am using again the shorthand $d^{w} \doteq[\text { the women } P]^{w}$ ).

a. [[The Jewish women $P]$ [ $\lambda x$ [[every Jewish man] $\lambda y[x$ related to $y]]]]$.

b. $\lambda w$. 【the women $P \rrbracket^{w} \in \operatorname{Dist}\left(\lambda x .\left[\llbracket\right.\right.$ every man $\rrbracket^{w} \ni \lambda y \cdot \operatorname{GEN}_{t}\left[\mathbf{C}^{w}(x, y, t)\right]\left[\llbracket\right.$ related $\left.\left.\left.\rrbracket^{w}(x, y, t)\right]\right]\right)$.

c. $\psi_{P}=\lambda w: Y E S(w) \vee \mathrm{NO}(w) . Y E S(w)$.

i. $Y E S(w)=1$ iff OMNI-RELATED ${ }^{w}(x)$ for every $x$ s.t. $x \leq_{\mathrm{AT}} d^{w}$,

ii. $N O(w)=1$ iff $\neg$ OMNI-RELATED ${ }^{w}(x)$ for every $x$ s.t. $x \leq_{\mathrm{AT}} d^{w}$,

iii.OMNI-RELATED ${ }^{w}(x) \doteq$

$\doteq[\text { every Jewish man }]^{w} \ni \lambda y \cdot \operatorname{GEN}_{t}\left[\mathbf{C}^{w}(x, y, t)\right]\left[[\text { related }]^{w}(x, y, t)\right]$.

Note that the logical equivalence in (45) holds, as can be verified in the same way as I did above for the equivalence (37). ${ }^{7}$ Note the following crucial difference between

\footnotetext{
${ }^{7}$ For the sake of explicitness, let me verify (45) explicitly. First, let me show that, if $\neg \beta(w)=$ 1 , then $\alpha(w)=1$. By contradiction, suppose $\alpha(w) \neq 1$. Hence there is at least one $\psi_{P}$ which is either true or undefined in $w$. This in turn means that there must exist at least a Jewish woman in $w$, say Mary, such that OMNI-RELATED ${ }^{w}($ Mary $)=1$, i.e. Mary is related to every Jewish man in $w$. Hence, $\beta(w)=1$, contradicting the hypothesis. Let me now show that, if $\alpha(w)=1$, then $\neg \beta(w)=1$. Consider an arbitrary Jewish woman in $w$, say Mary. Of course, there is some $P$ such that Mary $\leq_{\text {AT }}[\text { the women } P]^{w}$ (and, furthermore, such that $\psi_{P}$ asymmetrically entails $\varphi$ ). Note that $\psi_{P}$ must be false in $w$, because of the hypothesis $\alpha(w)=1$, i.e. $\psi_{P}(w)=0$. By virtue of the homogeneity presupposition, $\psi_{P}(w)=0$ iff for every $x$ such that $x \leq_{\mathrm{AT}}[$ the women $\left.P]\right]^{w}$, it happens that $\neg$ OMNI-RELATED ${ }^{w}(x)=0$, i.e. $x$ is not related to every Jewish man in $w$. Hence in particular the Jewish women Mary is not related to every Jewish man in $w$. Since this reasoning can be repeated for every other Jewish woman in $w$ besides Mary, then $\neg \beta(w)=1$. The logical equivalence in (45) is thus established.
} 
(45) and (37): in $\beta$ in (45), the existential quantifier over Jewish women has wide scope not only over the generic operator but also over 'every Jewish man' (whose denotation I am representing here just as $\forall_{y}$ ).

(45) For every world $w \in \mathscr{W}: \alpha(w)=1$ iff $\neg \beta(w)=1$.

$$
\begin{aligned}
& \text { a. } \alpha=\bigwedge\left\{\neg \psi_{P} \mid \psi_{P} \stackrel{\text { a }}{\rightarrow}_{\mathscr{W}} \varphi\right\} . \\
& \text { b. } \beta=\lambda w \cdot \exists_{x}\left[[[\operatorname{woman}]]^{w}(x) \wedge \forall_{y}\left[\operatorname{GEN}_{t}\left[\mathbf{C}^{w}(x, y, t)\right]\left[[[\text { related }]]^{w}(x, y, t)\right]\right]\right] .
\end{aligned}
$$

Let me now argue that the strengthened meaning $\operatorname{EXH}(\varphi)$ of $\varphi$ is the one in (46). i.e. that all the alternatives $\psi_{P}$ in (44b) are excludable wrt $\varphi$ in (43b). Under the assumption that there are no other relevant alternatives besides $\psi_{P}$ 's, it suffices to argue that $\operatorname{EXH}(\varphi)$ in (46) is not a logical contradiction. And by virtue of the equivalence in (45), it suffices to show that $\varphi \wedge \neg \beta$ is not a logical contradiction.

$$
\operatorname{EXH}(\varphi)=\varphi \wedge \underbrace{\bigwedge\left\{\neg \psi_{P} \mid \psi_{P} \stackrel{\mathrm{a}}{\rightarrow}_{\mathscr{W}} \varphi\right\}}_{\alpha} .
$$

To this end, consider the following possible world $w$ : there are only two Jewish men $a_{1}$ and $a_{2}$ and two Jewish women $b_{1}$ and $b_{2}$ in $w$; furthermore, the woman $b_{1}$ is related only to the man $a_{1}$ and the woman $b_{2}$ is related only to the man $a_{2}$. Note that $\varphi$ is true in such a world $w$ (with a judicious choice of the restrictive clause $\mathbf{C}$ ), since for every Jewish man there is a Jewish woman related to him. Note furthermore that $\beta$ is false in such a world $w$, since there is no Jewish woman related to every Jewish man. Thus, $\varphi \wedge \neg \beta$ is true in such a world $w$ and $\operatorname{EXH}(\varphi)=\varphi \wedge \neg \beta$. Note now that such a world $w$ could very well be compatible with the common knowledge $\mathscr{W}_{\text {ck }}$ in (28). Hence, the strengthened meaning $\operatorname{ExH}(\varphi)$ of $\varphi$ in (43b) is not a contradiction given common knowledge and the existential reading for the BPS of (42b) is not ruled out by (13). ${ }^{8}$

The modification suggested in (10) hasn't played any role in the paper so far; but it does play a crucial role to secure the latter result; here is why. Consider the alternatives in (47) for every $P$, which are obtained from (43a) by replacing 'Jewish women' by 'the Jewish women such and such' and furthermore 'every Jewish man' by 'some Jewish man'. Of course, (47) is a scalar alternative of $\varphi$ in (43c) for each $P$. It is easy to check that all these alternatives (47) can be negated consistently with $\varphi$ and that the strengthened meaning $\operatorname{EXH}(\varphi)$ so derived is a contradiction given the common knowledge $\mathscr{W}_{\text {ck }}$ in (28). Thus, we need to get the alternatives in (47) out of the set of excludable alternatives, in order to prevent the attested existential reading of the BPS in (42b) from being ruled out by (13).

$$
\left.\left.\lambda w \cdot[\text { the women } P]^{w} \in \operatorname{Dist}\left(\lambda x \cdot[[\operatorname{some} \operatorname{man}]]^{w} \ni \lambda y \cdot \operatorname{GEN}_{t}\left[\mathbf{C}^{w}(x, y, t)\right][[\text { related }]]^{w}(x, y, t)\right]\right]\right) .
$$

\footnotetext{
${ }^{8}$ Note furthermore that the approach developed so far predicts that the existential reading for the BPS of an i-predicate is available in the scope of a universal quantifier over individuals but not in the scope of a universal modal. The crucial difference between the two cases is that modals quantify over worlds and the scalar item [[the firemen such and such $]]^{w}$ has world-dependence built into it. This prediction is borne out: the BPS of a sentence such as 'Firemen must be tall' does not allow the existential reading.
} 
This problem is avoided by the modification in (10), since the alternatives in (47) are logically independent of $\varphi$ in (43b) and thus they cannot contribute to $\operatorname{ExH}(\varphi)$.

\subsection{Overt Q-Adverbs}

Contrary to s-predicates, i-predicates cannot occur with an overt Q-adverb (such a 'always' or 'often'), as shown by the different status of the two sentences (48). The oddness of sentence (48a) is particularly puzzling for my proposal, given that I am assuming that the corresponding fine sentence without overt 'always' contains its phonologically null counterpart GEN, as in (31).

(48) a. ?John is always tall.

b. John is always available.

a. Firemen are always tall.

b. A firemen is always tall.

Furthermore, Kratzer (1995) points out that sentence (48a) becomes fine if the definite 'John' is replaced by either a bare plural or an indefinite, as in (49). Let me argue that the oddness of (48a), repeated as $\varphi$ in (50a), can be accounted in the same way I accounted above for the oddness of the sentences (2). ${ }^{9}$ Consider the alternative $\psi$ in (50b) and assume that $\psi$ is a scalar alternative of $\varphi$.

a. $\varphi=$ ?John is always tall.

b. $\psi=$ John is GEN tall.

Of course, $\psi$ and $\varphi$ have the same meaning; nonetheless, they crucially have different presuppositions. As argued for example in von Fintel (1997), the covert generic operator GEN carries the homogeneity presupposition in (51), namely that the nuclear scope of GEN holds of each item in its restrictive clause or else is false for each such item. The homogeneity presupposition can be detected by means of negation: the sentence 'Italians don't eat raw fish' conveys that no single Italian eats raw fish, which is different from the plain meaning (namely that "it is false that every Italian eats raw fish") but does follow from the plain meaning plus the homogeneity presupposition. No such presupposition is carried by overt 'always'.

$\left[\operatorname{GEN}_{v_{1}, \ldots, v_{n}}\left[\operatorname{RC}\left(v_{1}, \ldots, v_{n}\right)\right]\left[\mathrm{NS}\left(v_{1}, \ldots, v_{n}\right)\right]\right]=\lambda w: Y E S(w) \vee N O(w) . Y E S(w)$

a. $Y E S(w)=1$ iff $\llbracket \mathrm{NS} \rrbracket\left(v_{1}, \ldots, v_{n}\right)$ for every $v_{1}, \ldots, v_{n}$ such that $\llbracket \mathrm{RC} \rrbracket\left(v_{1}, \ldots, v_{n}\right)$;

b. $N O(w)=1$ iff $\neg \llbracket \mathrm{NS} \rrbracket\left(v_{1}, \ldots, v_{n}\right)$ for every $v_{1}, \ldots, v_{n}$ such that $\llbracket \mathrm{RC} \rrbracket\left(v_{1}, \ldots, v_{n}\right)$.

Thus, the presuppositions of $\varphi$ and $\psi$ in (50) can be roughly spelled out as in (52).

a. $\varphi_{\text {prs }}=\mathscr{W}$.

b. $\psi_{\text {prs }}=Y E S \cup N O$

i. $Y E S=\{w \mid[\operatorname{tall}]](j, t)$, for every $t$ such that in $\left.^{w}(j, t)\right\}$;

ii. $N O=\left\{w \mid \neg[[\operatorname{tall}]](j, t)\right.$, for every $t$ such that $\left.\operatorname{in}^{w}(j, t)\right\}$.

\footnotetext{
${ }^{9}$ This proposal is an elaboration of a suggestion by Danny Fox.
} 
Of course, $\psi_{\text {prs }}$ asymmetrically entails $\varphi_{\text {prs }}$. Thus, the strengthened presupposition of $\varphi$ boils down to $\operatorname{EXH}\left(\varphi_{\text {prs }}\right)=\varphi_{\text {prs }} \wedge \neg \psi_{\text {prs }}=\neg \psi_{\text {prs }}$. This strengthened presupposition is a contradiction given common knowledge $\mathscr{W}_{\text {ck }}$ in (28), since $\mathscr{W}_{\text {ck }}$ entails $\psi_{\text {prs }}$. Thus, the oddness of sentence (48a) follows from (23).

The situation is quite different in the case in which 'John' is replaced by a bare plural or an indefinite as in (49), repeated below as $\varphi$ in (53a), together with the alternative $\psi$ in $(53 \mathrm{~b})$.
a. $\varphi=$ Firemen / a fireman are / is always tall.
b. $\psi=$ Firemen $/$ a fireman are / is GEN tall.

In the case of $\varphi$ and $\psi$ in (53), the Q-adverbs GEN and 'always' are quantifying both over times and over firemen (see Diesing (1992) or Chierchia (1995) for discussion). Thus, the homogeneity presupposition for $\psi$ yields (54b) in this case.

a. $\varphi_{\text {prs }}=\mathscr{W}$.

b. $\psi_{\text {prs }}=Y E S \cup N O$
i. YES $=\left\{w \mid[\operatorname{tall} 1](x, t)\right.$, for every $x, t$ such that $\left[[\text { firemen }]^{w}(x)\right.$ and $\left.\mathbf{i n}^{w}(x, t)\right\}$
ii. $N O=\{w \mid \neg[$ tall $]](x, t)$, for every $x, t$ such that $[[\text { firemen }]]^{w}(x)$ and $\left.\mathbf{i n}^{w}(x, t)\right\}$.

Again, $\psi_{\text {prs }}$ asymmetrically entails $\varphi$ and the strengthened presupposition of $\varphi$ boils down to $\operatorname{EXH}\left(\varphi_{\text {prs }}\right)=\varphi_{\text {prs }} \wedge \neg \psi_{\text {prs }}=\neg \psi_{\text {prs }}$. But this time, $\neg \psi_{\text {prs }}$ is not a contradiction given the common knowledge $\mathscr{W}_{\text {ck }}$ in (28), since there are of course worlds compatible with common knowledge where $\psi_{\text {prs }}$ is false because some firemen are tall while others are not. The felicity of sentences (49) is thus predicted. The account furthermore predicts that sentences of the type of (49) should sound odd in cases where common knowledge entails that the individuals denoted by the BPS are homogeneous with respect to the property denoted by the i-predicate. This prediction seems to be borne out by the contrast in (55), pointed out to me by Danny Fox (I am assuming that 'speak' is construed as an i-predicate here).

a. Hebrew speakers speak a vary difficult language.

b. ?Hebrew speakers always speak a very difficult language.

\section{Conclusions}

The following two claims have been defended in the recent literature. First, that the theory of scalar implicatures must be grammatical rather than pragmatic in nature. Second, that a grammatical theory of scalar implicatures can be used to account for facts which have a grammatical flavor. See for instance the account for the intervention effect with NPIs in Chierchia (2004). In this paper, I have tried to contribute to this line of research, by means of the following two claims. In Section 1, I have presented one more argument in favor of a grammatical rather than pragmatic theory of implicatures, namely the BH (12). In Section 2, I have shown that some properties of i-predicates, which have been so far accounted for by means of specific 
grammatical assumptions, naturally follow from a theory of implicatures endowed with the $\mathrm{BH}$.

\section{References}

Chierchia, Gennaro: 1995, 'Individual-Level Predicates as Inherent Generics', in G. N. Carlson and F. J. Pelletier (eds.), The Generic Book, 125-175. The Univ. of Chicago Press.

Chierchia, Gennaro: 1998, 'Reference to Kinds Across Languages', Natural Language Semantics 6, 339-405.

Chierchia, Gennaro: 2004, 'Scalar Implicatures, Polarity Phenomena and the Syntax/Pragmatics Interface', in A. Belletti (ed.), Structures and Beyond. Oxford University Press, Oxford.

Diesing, Molly: 1992, Indefinites. Cambridge, Mass.: MIT Press.

von Fintel, Kai: 1997, 'Bare Plurals, Bare Conditionals, and Only', Journal of Semantics 14, 1-56.

Fodor, Janet Dean: 1970, The Linguistic Description of Opaque Contexts, $\mathrm{PhD}$ dissertation, MIT.

Fox, Danny: 1995, 'Economy and Scope', Natural Language Semantics 3, 283341.

Fox, Danny: 2006, 'Free Choice and the Theory of Scalar Implicatures'. MIT ms.

Fox, Danny and Martin Hackl: 2005, 'The Universal Density of Measurment'. ms.

Gajewski, Jon: 2005, Neg-Raising: Polarity and Presupposition, PhD dissertation, MIT.

Hawkins, John A.: 1991, 'On (In)Definite Articles: Implicatures and (Un)Grammaticality Prediction', Journal of Linguistics 27, 405-442.

Heim, Irene: 1991, 'Artikel und Definitheit', in A. von Stechow and D. Wunderlich (eds.), Semantik: Ein Internationales Handbuch der Zeitgenössischen Forschung, 487-535. De Gruyter: Berlin.

Kratzer, Angelika: 1995, 'Stage-Level and Individual-Level Predicates', in G. N. Carlson and F. J. Pelletier (eds.), The Generic Book, 125-175. The Univ. of Chicago Press.

Maienborn, Claudia: 2004, 'A Pragmatic Explanation of the Stage Level/Individual Level Contrast in Combination with Locatives', in B. Agbayani, V. Samiian, and B. Tucker (eds.), Proceedings of the Western Conference of Linguistics (WECOL), 158-170. Fresno: CSU. volume 15.

Percus, Orin: 2001, 'Pragmatic Constraints on (Adverbial) (Temporal) Quantification'. ms.

Percus, Orin J.: 1997, Aspects of 'A', PhD dissertation, MIT.

Sauerland, Uli: 2003a, 'A New Semantics for Number', in Proceedings of SALT13. Sauerland, Uli: 2003b, 'Implicated Presuppositions', in Proceedings of "Polarity, Scalar Phenomena, Implicatures”, Univ. of Milano Bicocca, June 18-20 2003. 